\title{
TTR
}

Traduction, terminologie, rédaction

\section{Traduire la banlieue : défis et obstacles}

\section{Ilaria Vitali}

Volume 31, numéro 1, 1er semestre 2018

Traduire la banlieue : problématiques, enjeux, perspectives

Translating the Banlieue: Issues, Challenges, Perspectives

URI : https://id.erudit.org/iderudit/1062552ar

DOI : https://doi.org/10.7202/1062552ar

Aller au sommaire du numéro

\section{Éditeur(s)}

Association canadienne de traductologie

ISSN

0835-8443 (imprimé)

1708-2188 (numérique)

Découvrir la revue

Citer cet article

Vitali, I. (2018). Traduire la banlieue : défis et obstacles. TTR, 31(1), 193-220.

https://doi.org/10.7202/1062552ar

\section{Résumé de l'article}

Dans une étude assez récente, Gisèle Sapiro (2012) mettait l'accent sur les obstacles à la traduction dans le domaine littéraire. En effet, si l'on parle souvent avec enchantement (et parfois de façon naïve) du " dialogue entre les cultures " prôné par la traduction littéraire, on s'intéresse moins aux conditions et aux obstacles que ce dialogue peut poser. La question est pourtant primordiale, surtout lorsqu'on étudie la traduction des romans d'écrivains dits " de banlieue ". De par leur spécificité, ces romans mobilisent des rapports de force d'ordre linguistique, social et culturel. En prenant appui sur une étude de cas constituée par la traduction italienne des romans de Saphia Azzeddine, cet article cherche à sonder les défis et obstacles posés par la traduction, que l'on peut diviser en deux catégories enchevêtrées et interdépendantes : les obstacles internes, liés aux difficultés traductives posées par l'emploi et le détournement de l'argot des cités; les obstacles externes, qui concernent les politiques éditoriales ainsi que les facteurs sociaux et économiques relatifs à la circulation des oeuvres dans le marché contemporain.
Ce document est protégé par la loi sur le droit d'auteur. L'utilisation des services d’Érudit (y compris la reproduction) est assujettie à sa politique d'utilisation que vous pouvez consulter en ligne.

https://apropos.erudit.org/fr/usagers/politique-dutilisation/ 


\title{
Traduire la banlieue : défis et obstacles
}

\author{
Ilaria Vitali \\ Università di Macerata
}

\begin{abstract}
Résumé
Dans une étude assez récente, Gisèle Sapiro (2012) mettait l'accent sur les obstacles à la traduction dans le domaine littéraire. En effet, si l'on parle souvent avec enchantement (et parfois de façon naïve) du «dialogue entre les cultures» prôné par la traduction littéraire, on s'intéresse moins aux conditions et aux obstacles que ce dialogue peut poser. La question est pourtant primordiale, surtout lorsquon étudie la traduction des romans d'écrivains dits «de banlieue». De par leur spécificité, ces romans mobilisent des rapports de force d'ordre linguistique, social et culturel. En prenant appui sur une étude de cas constituée par la traduction italienne des romans de Saphia Azzeddine, cet article cherche à sonder les défis et obstacles posés par la traduction, que l'on peut diviser en deux catégories enchevêtrées et interdépendantes : les obstacles internes, liés aux difficultés traductives posées par l'emploi et le détournement de l'argot des cités; les obstacles externes, qui concernent les politiques éditoriales ainsi que les facteurs sociaux et économiques relatifs à la circulation des œuvres dans le marché contemporain.
\end{abstract}

Mots-clés: banlieue, langue des jeunes, argot des cités, traductologie, Saphia Azzeddine

\begin{abstract}
In a recent study, Gisèle Sapiro (2012) emphasised the obstacles to translation in the field of literature. Indeed, if we often speak with delight (and at times a certain naivety) of the "dialogue between cultures" advocabed by literary translation, less attention is paid to the conditions and obstacles that are involved in this dialogue. Yet, this issue is of utmost importance, especially when dealing with the translation of novels written by the socalled "banlieue" writers. By their very specificity, these novels mobilise power relations on the linguistic, social and cultural levels. On the basis of a case study of the Italian translations of Saphia Azzeddine's novels, this article aims at revealing the challenges and obstacles posed by translation, which can be divided into two intertwined and interdependent categories: internal obstacles, linked to translation difficulties raised by the use and misappropriation of banlieue slang; external obstacles due to editorial poli-
\end{abstract}


cies as well as social and economic factors relating to the circulation of books in the modern-day market.

Keywords: banlieue, youth slang, argot des cités, translation studies, Saphia Azzeddine

\section{Introduction}

À la suite des émeutes qui ont touché les cités françaises à partir de novembre 2005, la banlieue a eu droit à un regain d'intérêt du point de vue socioculturel aussi bien que littéraire. Il suffit de penser aux ouvrages de Mabrouck Rachedi, Samuel Benchetrit ou Rachid Djaïdani, pour ne citer que quelques exemples. Ces auteurs nous invitent à découvrir un univers suburbain craint, fantasmé et souvent mal connu, et s'attachent à montrer un visage différent de «la zone» par une posture engagée à plusieurs niveaux ${ }^{1}$. Aujourd'hui, l'image de ces écrivains est liée non seulement aux thèmes qu'ils abordent - exclusion sociale, émeutes urbaines, discrimination raciale - mais aussi au langage qu'ils emploient, qui puise de manière créative dans cette variété de «français des cités» caractérisée par divers procédés formels et sémantiques; on pense notamment à la déformation verlanesque ainsi qu'à l'utilisation de mots issus du vieil argot et d'emprunts à d'autres langues, notamment extra-européennes (Goudaillier, 1997, p. 17).

Ce procédé de dynamisation - et «dynamitation »-linguistique et littéraire fortifie les textes de ces auteurs, mais il contribue en même temps à les rendre apparemment «intraduisibles». Pourtant, ces écrivains dépassent de plus en plus les frontières de l'Hexagone. Une recherche croisée dans l'Index Translationum (UNESCO, s.d.) ${ }^{2}$, les bases de données du site Limag (Bonn et Coordination internationale des chercheurs sur les littératures du Maghreb, 1998) et Google Books permet en effet de constater une flambée des traductions depuis 2005. Tout traducteur qui s'approche des romans dits «de banlieue» aura toutefois affaire à des textes qui demandent un effort interprétatif important et qui résistent - du moins à première vue - aux tentatives de traduction. Comment traduire une

1. Des études récentes ont envisagé, sous des angles divers, l'engagement des hommes et des femmes en banlieue (cf. Paquot, 2008; Turpin, 2012; Carpenter et Horvath, 2014; Vitali, 2014; Wallon, 2016).

2. L'Index Translationum n'est malheureusement pas mis à jour de manière régulière et homogène; il faut donc se servir d'autres outils et bases de données pour obtenir des résultats plus fiables et plus complets. 
réalité et un langage si spécifiques? Doit-on préférer une traduction étrangéisante qui préserve les realia (Vlahov et Florin, 1986) ou opérer une adaptation transculturelle? Quels sont les auteurs traduits, et de quelle manière les traductions influencent-elles, hors de France, la compréhension et la représentation des banlieues? Et encore, qui choisit ces textes (les éditeurs, les traducteurs, les universitaires...) et quel accueil leur réserve-t-on? Autant de questions qui illustrent les enjeux auxquels sont confrontés les chercheurs dans ce champ qui reste encore à défricher.

Dans une étude publiée en 2012, Gisèle Sapiro mettait l'accent sur les obstacles économiques et culturels à la traduction dans le domaine littéraire. En effet, si l'on parle souvent avec enchantement (et parfois de façon naïve) du «dialogue entre les cultures» prôné par la traduction littéraire, on s'intéresse moins aux conditions et aux difficultés que ce dialogue peut poser. La question est pourtant primordiale, surtout lorsqu'on s'intéresse à la traduction des romans dits «de banlieue». De par leur spécificité, ces ouvrages mobilisent, comme on le verra, des rapports de force d'ordre linguistique, social et culturel qui déterminent les choix éditoriaux ainsi que les postures des traducteurs.

\section{Méthodologie et corpus}

En partant des prémisses exposées dans l'introduction, cet article cherche à sonder les défis et les obstacles à la traduction des œuvres de la littérature de banlieue. J'entends par «littérature de banlieue» l'ensemble des fictions écrites dont la banlieue constitue non seulement le cadre de l'action, mais également le thème principal de l'intrigue ${ }^{3}$. On peut classer ces défis et obstacles en deux catégories : d'une part, ceux qui sont internes au texte et qui relèvent des difficultés traductives que soulève l'usage créatif de la «langue des cités»; d'autre part, ceux qui sont externes au texte et qui concernent les politiques éditoriales, l'idéologie et la culture, ainsi que les facteurs sociaux et économiques liés à la circulation des œuvres dans le marché contemporain. Ces deux catégories sont enchevêtrées et interdépendantes. La méthodologie d'analyse mise de l'avant s'inspire des travaux de Gisèle Sapiro (2008 et 2012), quoiqu'elle ait été adaptée au contexte d'une recherche plus ciblée -

3. Je n'approfondirai pas ici la question des classements et des étiquettes, maintes fois abordée. À ce sujet, on pourra consulter notamment Hargreaves (2014, entre autres), Reeck (2011), Horvath (2016) et Vitali (2011 et 2014). 
les romans abordant la problématique des banlieues - et donc plus restreinte. Le tout sera illustré d'exemples empruntés au contexte de l'Italie, où je travaille en tant que traductrice ${ }^{4}$ et universitaire.

Pour essayer de répondre aux questions posées dans l'introduction, je me référerai à la traduction des romans de Saphia Azzeddine, romancière d'origine franco-marocaine née en 1979. Je propose de suivre le parcours italien de deux romans édités en France par Léo Scheer, Mon père est femme de ménage (2009) et La MecquePhuket (2010), à partir de la sélection des ouvrages de la part des éditeurs jusqu'à leur traduction, production et commercialisation ${ }^{5}$. Comme on le verra, ces deux romans racontent le même univers des marges : ils ont pour narrateurs des jeunes gens de la banlieue parisienne qui essayent de se frayer un chemin dans un monde difficile et assez hostile, bien que le ton de la narration demeure enjoué et souvent poétique.

En ce qui concerne le contexte éditorial, il n'est pas anodin de souligner que l'Italie demeure le principal acheteur de droits français. Comme le montrent les statistiques du Syndicat national de l'édition de France, depuis le début du millénaire, l'Italie, l'Espagne et l'Allemagne sont dans l'ordre les principaux acheteurs de droits français (les données de 2016-2017 indiquent respectivement 1088 cessions pour l'Italie, 1003 pour l'Espagne et 815 pour l'Allemagne). À ces trois pays européens s'est ajoutée récemment la Chine, qui a montré un intérêt croissant pour la France ces dernières années (plus de 2100 titres ont été cédés à des éditeurs chinois en 2016). Selon les rapports du Syndicat national de l'édition de France et du Bureau international de l'édition française, ce n'est toutefois pas le domaine littéraire qui intéresse le plus les éditeurs chinois, mais plutôt celui des sciences humaines, la littérature française étant perçue comme «élitiste» et difficile d'accès.

\footnotetext{
4. J'ai une certaine expérience dans la traduction des romans ou, plus en général, des textes en prose centrés sur la problématique des banlieues. J'ai traduit, entre autres auteurs, Saphia Azzeddine, Samuel Benchetrit, Rachid Djaïdani, Mabrouck Rachedi et le collectif d'écrivains "Qui fait la France?».

5. Je suis bien consciente qu'il ne s'agit que d'une étude de cas, avec toutes les limites que cela comporte. Je l'ai choisie après avoir procédé à une analyse de large spectre, parce qu'elle me semble constituer un cas représentatif dans le domaine envisagé. Des exemples ponctuels tirés de romans d'autres écrivains ainsi que des renvois aux traductions allemande et espagnole des romans d'Azzeddine viendront enrichir ma réflexion.
} 
Ces tendances générales se reflètent-elles dans la traduction des romans traitant spécifiquement des banlieues? Les données encore lacunaires et morcelées ne permettent pas d'avoir des statistiques complètes sur l'ensemble des traductions réalisées dans le monde par rapport à notre domaine de recherche. Si on se réfère à notre étude de cas, on notera en tout cas que les romans de Saphia Azzeddine ne sont effectivement traduits qu'en Italie, en Allemagne et en Espagne.

\section{Les choix éditoriaux}

Pour un éditeur, une traduction est au départ un travail en perte : achat des droits et rémunération du traducteur s'ajoutent aux frais de publication. Comme la traductrice Françoise Bouillot le résume brillamment, «[s]ont en jeu : une somme de mots, une somme de temps, une somme d'argent» (2010, p. 88). Gisèle Sapiro a montré que nombre d'éditeurs refusent désormais d'envisager un projet de traduction sans la perspective d'une aide financière (2012, p. 33) et que tout travail de traduction implique un certain volontarisme de la part des nombreux acteurs de la chaîne du livre, en commençant par le traducteur, notamment lorsqu'on a affaire à des éditeurs indépendants.

Un premier obstacle à la publication des textes en traduction est donc constitué par le coût du projet, ce qui explique l'extrême prudence des éditeurs dans leurs choix. Des romans liés particulièrement à la situation et aux interrogations d'un pays, comme c'est le cas pour la question des banlieues, peuvent ne pas intéresser d'autres pays. Le premier défi concerne ainsi la publication des romans de notre domaine, qui peut se révéler assez ardue, sauf lorsque l'intérêt médiatique pour un évènement tel que les émeutes en banlieue transcende les frontières nationales. Au-delà de ces événements d'intérêt circonstanciel, le processus de choix éditorial est très réfléchi par rapport aux titres qui ne nécessitent pas de traduction (cf., entre autres, Sapiro, 2012). C'est sans doute pour cette raison que les éditeurs, surtout ceux de petite ou moyenne taille, peuvent s'adresser à des spécialistes dans le secteur. À ce sujet, l'influence des voix provenant du monde universitaire ne doit pas être sousestimée. La circulation internationale des ouvrages dans le champ de recherche envisagé se fait couramment par le biais de passeurs universitaires et souvent sous la forme d'un effet «boule de neige». Selon Anne-Claire Collier (2007), cette même dynamique dépasse 
le domaine de la fiction pour traverser celui des ouvrages critiques dans un contexte "postcolonial ${ }^{6}$. Cela s'avère aussi dans le cas des écrivains qui traitent des banlieues, eux-mêmes souvent proches de ceux qu'Abdourahman A. Waberi (1998) définit comme auteurs de la «postcolonie $»^{7}$.

Tous les ouvrages que j'ai traduits ont été proposés par moimême aux éditeurs avec lesquels j'ai travaillé ${ }^{8}$. Je l'ai fait de ma propre initiative au début de ma carrière et j'ai ensuite été sollicitée par les éditeurs. Dans mon étude de cas, qui concerne les romans de Saphia Azzeddine, j'ai été sollicitée une première fois en 2007 par Giulio Perrone, maison d'édition indépendante fondée à Rome en 2005. La traduction d'Azzeddine entrait dans le cadre d'un projet plus large que l'éditeur m'avait commandé pour promouvoir des auteurs français émergents. Parmi les propositions, les romans de Rachid Djaïdani, Samuel Benchetrit et Saphia Azzeddine avaient été retenus par la directrice éditoriale, Mariacarmela Leto. Ces traductions ont fait l'objet d'une aide financière de la part du ministère français des Affaires étrangères et du développement international, par le biais des programmes d'aide à la publication d'œuvres françaises en Italie. Pour cet éditeur, j'ai traduit le deuxième roman d'Azzeddine, Mon père est femme de ménage (2009; Mio padre fa la donna delle pulizie, 2011). Cet ouvrage, qui raconte l'adolescence de Polo dans une banlieue défavorisée de l'île de France avec un père nettoyeur et rêvant d'une place au soleil, avait été apprécié par le comité de lecture moins pour l'intérêt sociologique de ses contenus que pour le style de l'auteure, teinté d'humour et de poésie. La narration percutante, rythmée et captivante des aventures de Polo, passionné de littérature qui cherche dans des livres immortels les mots pour sortir de son milieu, épousait parfaitement les enjeux de la collection Hinc de Perrone, qui accorde une large attention au style des auteurs, notamment de jeunes écrivains prometteurs qui en sont souvent à leurs débuts et

6. Collier souligne d'ailleurs «toute l'ambiguïté de la place des éditeurs dans la traduction des études postcoloniales [...] et le rôle qu'ils ont pu jouer (ou auquel ils ont été assignés) lors de cette circulation internationale des textes» (2015, p. 56).

7. À ce sujet, on lira avec profit Lapeyronnie (2005), qui envisage la banlieue comme un «théâtre colonial».

8. Bien évidemment, ce ne sont pas tous les ouvrages que j'ai proposés qui ont été retenus pour publication. 
qui ont des carrières fulgurantes (qui se mesurent, entre autres, aux reconnaissances et prix prestigieux comme le Premio Strega ou le Campiello, ou à des publications chez des éditeurs de plus grand renom $)^{9}$. Comme le relève Chiara Elefante, il s'agit d'un éditeur, et particulièrement d'une collection, «qui ne suit pas les tendances de lecture déjà existantes, mais en anticipe d'autres, celles des lecteurs les plus exigeants» $(2015$, n.p.).

Quelques années plus tard, en 2014, j’ai été contactée par la maison d'édition indépendante Il Sirente, fondée en 1998 sur les versants du mont dont elle porte le nom. D’abord spécialisée dans l'édition scientifique, la maison Il Sirente est plus marquée du point de vue politique que Perrone. Elle s'est distinguée par la publication de textes de sciences sociales, politiques et juridiques, avant de se faire connaître dans le domaine de la fiction en 2007, notamment par la création d'une collection-phare nommée Altriarabi, consacrée aux écrivains arabophones. Il Sirente souhaitait en 2014 créer une collection consacrée aux écrivains d'origine arabe nés ou ayant grandi en Europe et écrivant dans une langue européenne, avec une attention particulière au phénomène de la marginalisation. Cette collection, nommée Altriarabi migrante, a été conçue comme la «sœur» de la collection Altriarabi. Le projet, financé par le programme «Europe Créative» de l'Union européenne, a permis la publication de titres de backlist ${ }^{10}$ de plusieurs langues et pays européens ${ }^{11}$. Le but du projet est bien expliqué par la directrice éditoriale, Chiarastella Campanelli :

Dopo 7 anni dalla nascita del progetto editoriale Altriarabi, è sorta l'esigenza di raccogliere anche le voci degli europei con origine araba. Un canto proveniente dal sud e un contro-canto proveniente dal nord, entrambi affacciati sulle rive del mar mediterraneo - «al bahr al abyad al mutawassit», il mare bianco di mezzo - per dialogare, confrontarsi, guardarsi. (2015, p. II)

[Sept années après la naissance du projet éditorial Altriarabi,

9. Je pense, par exemple, à Paolo di Paolo, Giuseppe Aloe ou Giulio Perrone lui-même.

10. On entend par backlist l'ensemble des titres publiés par un éditeur et toujours disponibles; la backlist s'oppose à la frontlist, qui concerne les nouvelles parutions. 11. Voici quelques romans qui ont été traduits de langues européennes autres que le français et qui ont été publiés dans la collection entre 2015 et 2017 : The Boy from Aleppo who painted the War de Sumia Sukkar; De autist en de postduif de Rodaan Al Gaalidi; Der falsche Inder de Abbas Khider; Out of it de Selma Dabbagh. 
l'exigence est née de recueillir aussi les voix des Européens d'origine arabe. Un chant provenant du sud et un contre-chant provenant du nord, les deux ouverts sur les rives de la mer Méditerranée - «al bahr al abyad al mutawassit», la mer blanche du milieu - pour dialoguer, se confronter, se regarder.] (ma trad.)

Pour des raisons historiques, la France est le pays d'Europe qui compte la communauté d'origine maghrébine la plus importante $^{12}$. Après avoir traduit pour Il Sirente Les Mohamed de Jérôme Ruillier, la première bande dessinée à traiter de manière globale le phénomène de l'immigration maghrébine en France, le roman d'Azzeddine La Mecque-Phuket (2010), qui propose une réflexion enjouée sur la vie quotidienne de la communauté d'origine maghrébine en banlieue parisienne, m'a paru une bonne proposition à avancer. Le roman montre bien les différences entre la génération des immigrés primo-arrivants, encore tournée vers sa communauté d'origine, et celle de leurs enfants nés en France, tournée vers de nouveaux horizons. La narratrice et héroïne du roman, la jeune Fairouz, travaille durement pour offrir à ses parents un voyage à la Mecque, tout en étant tentée par d'autres destinations... Entre la quête de ses origines arabes et l'adhésion aux codes de la société française, elle finira par choisir un "troisième espace», représenté ici par Phuket, ne serait-ce que le temps de vacances.

Ma traduction du roman, parue sous le titre La Mecca-Phuket, a été publiée en 2017, après une phase d'achat de droits assez longue et complexe. Dans ce cas, au-delà de l'appréciation du style de l'écrivaine, c'est surtout le contenu du roman qui a guidé le choix de l'éditeur italien, qui souhaitait bâtir une collection cohérente et solide par rapport aux enjeux posés. Dès le début, les deux romans d'Azzeddine, publiés en France par le même éditeur, ont donc été appréciés pour des raisons différentes par les éditeurs italiens, ce qui s'est reflété dans la suite du processus éditorial.

L'étape cruciale qui suit l'achat des droits est constituée par la traduction elle-même qui, dans le cas de notre domaine de recherche, soulève plusieurs questions d'ordre non seulement linguistique, mais également éthique et idéologique.

12. Selon les statistiques de l'agence de l'Union européenne Frontex, les Maghrébins et leurs descendants en France sont environ 8 millions (cf. <http://frontex. europa.eu>); à ce sujet, on pourra aussi consulter le site de la Cité nationale de l'histoire de l'immigration à l'adresse suivante: <http://www.histoireimmigration.fr >. 


\section{Défis internes aux textes et postures traductives}

On l'a vu, l'image des écrivains traitant de la banlieue est de nos jours liée non seulement à la narration qu'ils font de ces territoires en souffrance, mais aussi au langage très particulier qu'ils emploient, langage qui puise avec créativité dans cette variété de français que certains tels Bernard Conein et Françoise Gadet (2000), et MarieMadeleine Bertucci et Daniel Delas (2004), considèrent comme un nouvel avatar de l'argot traditionnel ou de la langue populaire, alors que d'autres, comme Jean-Pierre Goudaillier (1997), y voient une variété à part entière, caractérisée surtout par la place de choix qu'y occupent les emprunts aux langues extra-européennes, reflet d'une immigration importante ${ }^{13}$. Aux yeux de certains spécialistes, utiliser les termes «banlieue» ou «cité» pour définir ce langage risque de marquer d'un sceau les locuteurs en question. Loin d'y voir moi-même une limitation, ou pire une ghettoïsation, je précise que j'entends l'emploi littéraire de ce langage comme une marque de créativité et de renouvellement stylistique, ainsi que d'une maîtrise exceptionnelle des différents procédés de création formels et sémantiques qui sont à l'œuvre dans ce langage dont les écrivains sont bien conscients (à la différence, sans doute, des simples locuteurs). Je dis bien "emploi littéraire de ce langage», car les auteurs qui l'utilisent ne se limitent aucunement à reproduire la variété diastratique et diatopique en usage dans les cités pour obtenir un effet de réalisme, ce qui serait dépourvu d'intérêt. Au contraire, ils en tirent profit en tout état de cause, au point d'en narrativiser les procédés, en les exploitant comme ressort narratif outre que comme trait stylistique, en émaillant leurs textes de nombreuses réflexions métalangagières (Vitali, 2015). Le traducteur de ces textes sera donc confronté non seulement à la présence de tournures argotiques et populaires, mais surtout à leur «mise en scène».

Quelle posture adopter, donc, pour reproduire la complexité de cette stratification langagière? Cela dépend du traducteur, certes, mais aussi du contexte éditorial, car aucune traduction publiée

13. Pour reprendre les mots de Goudaillier : «Si l'on prend en considération ce qui s'est passé depuis cent ans environ pour l'argot traditionnel, [...] une différence fondamentale doit être notée par rapport à ce que l'on constate aujourd'hui sur le terrain : de nos jours les épices importées et instillées dans la langue française sont de plus en plus fréquemment empruntées à des langues étrangères. Même si l'argot traditionnel a su s'alimenter de termes étrangers, il le faisait dans des proportions moins importantes» (Goudaillier, 1998, p. 13). 
n'existe a priori de ce contexte. Le panorama éditorial actuel est sans aucun doute très varié, mais il n'en reste pas moins que deux macrotendances s'observent : la neutralisation, c'est-à-dire une «domestication» qui vise en premier lieu la compréhension du lecteur (tendance normalement suivie par les groupes éditoriaux à grands tirages); l'étrangéisation, c'est-à-dire la tentative de garder intacte au possible le palimpseste linguistique, en ajoutant éventuellement un apparat paratextuel - glossaire, notes de bas de page, préface ou postface - tout en gardant à l'esprit qu'il est destiné à un public non spécialisé (tendance suivie par les éditeurs indépendants, à petits tirages). ${ }^{14}$

Chiara Elefante (2015) l'a montré en prenant en exemple les traductions italiennes de Faïza Guène et du collectif «Qui fait la France? ${ }^{15}$, publiées respectivement chez Mondadori (le plus important groupe éditorial italien, dans une collection grand public appelée Omnibus) et chez Stampa Alternativa (éditeur indépendant caractérisé pour son engagement politique et idéologique, dans une collection aux allures provocatrices intitulée Eretica). Les stratégies adoptées par les traducteurs, en accord avec les éditeurs et/ou les directeurs de collections, reflètent deux postures radicalement différentes: dans le premier cas, les traductions destinées au grand public sont naturalisantes et réduisent l'écart entre texte source et langue cible; dans le second cas, les traductions soutenues par les éditeurs indépendants privilégient une posture plus «éthique», pour reprendre la terminologie d'Antoine Berman (1985), qui préserve au possible la superposition des langues et des cultures ainsi que les réseaux vernaculaires sous-jacents.

Ce schéma se révèle opératoire dans des réalités culturelles et linguistiques autres que l'Italie. Regina Keil-Sagawe, célèbre traductrice allemande d'auteurs francophones d'origine maghrébine, souligne entre autres jusqu'à quel point les éditeurs peuvent effacer

14. Je précise qu'il s'agit de tendances et que plusieurs nuances sont possibles entre la naturalisation et l'étrangéisation, pour reprendre les termes bien connus de Venuti (1998).

15 Son étude a le mérite d'avoir dépassionné l'éternel débat entre sourciers et ciblistes dans le domaine en question, en considérant d'abord les contextes éditoriaux qui ont rendu possibles les traductions et en les analysant en conséquence, sans les considérer comme des produits existant a priori ou en dehors du système éditorial. 
le style d'un écrivain "périphérique» en fonction du public ciblé :

le cas de figure le plus frappant reste sans doute Yasmina Khadra qui, depuis la parution-choc de Morituri, son policier islamiste, en 1999, en est à son quinzième roman traduit et à son cinquième éditeur allemand. Ayant traduit la quasi-totalité de ce corpus, j'ai pu constater que ce qui est considéré comme splendeur ou misère de son style, selon l'optique du critique, c'est-à-dire un pathos et une exubérance verbale hors pair, a été progressivement supprimé par les correcteurs relisant mes traductions, d'une maison d'édition à l'autre, au fur et à mesure que Khadra devenait un auteur de best-sellers. (2017, p. 196)

Keil-Sagawe ajoute que ces propos peuvent être étendus à d'autres auteurs «migrants» ou "périphériques». Or, il est clair que si la traduction qui neutralise l'«exubérance verbale» demeure plus lisible pour le lecteur du texte cible, il en résulte que toute la symbolique sous-jacente à sa présence est perdue ou amoindrie, car l'usage du français des cités dans les textes en question ne se réduit pas à un simple effet de réalisme, mais comporte un projet poétique précis, voire des aspects d'ordre social et idéologique (cf. Vitali, 2015). De plus, les aspirations des personnages représentés dans les romans passent souvent par leur usage du/des langage(s), ce qui est d'ailleurs rendu explicite par les nombreux passages métalinguistiques. Cela dit, en tant que traductrice, je sais bien, tout comme Keil-Sagawe, que

le clivage est parfois grand entre la théorie, préconisant, dans la filiation de Berman, la «survie de l'étrangeté du texte de départ», et la pratique traductive au quotidien, influencée par les us et coutumes du système éditorial, favorisant l'adaptation de la traduction à l'horizon d'attente présumé du public. (2017, p. 196)

Dans le cas de la traduction de Saphia Azzeddine, les problématiques diastratiques principales étaient constituées sur le plan syntaxique par un relâchement du langage frisant parfois les fautes et sur le plan lexical par la présence de termes argotiques et verlanisés. Comme je l'ai mentionné ailleurs (cf. Vitali, 2011), l'italien ne peut pas compter sur un argot commun ni sur un langage à clé comparable au verlan. Il fallait donc trouver des solutions qui puissent rendre compte de la déformation, de l'insoumission aux contraintes, voire de "l'insubordination» langagière choisies par l'auteure. Pour recréer ces aspects dans leur ensemble, j’ai puisé dans les couches de 
l'italien néo-standard et sub-standard (Berruto, 2012) ${ }^{16}$, notamment pour la traduction des dialogues qui reproduisaient la spontanéité d'un langage oral et non surveillé. Au niveau morphosyntaxique, j’ai opté pour une simplification du système verbal, en remplaçant le subjonctif par l'indicatif, construction fautive typique de l'oralité :

J'ai fait comme si je savais. (Azzeddine, 2010, p. 172)

Ho fatto come se lo sapevo. (Azzeddine, 2017, p. 104)

J'ai également exploité le «che» (que) polyvalent, typique du langage relâché, comme dans les exemples suivants :

Alors dis-moi le tien que je vois s'il est mieux? (Azzeddine, 2010, p. 149)

Allora dimmi il tuo che vedo se è meglio. (Azzeddine, 2017, p. 89)

ça fait genre t'es occupée (Azzeddine, 2009, p. 158)

sembra come che sei occupata (Azzeddine, 2011, p. 106)

Toujours au sujet du «che» (que), je l'ai employé également pour introduire certaines phrases interrogatives, selon un usage populaire typique du centre de l'Italie et de plus en plus répandu dans les médias (Berruto, 2012, p. 235). J'ai réservé cette tournure au personnage de Kalsoum, la sœur de l'héroïne Fairouz dans La MecquePhuket, pour caractériser son discours de locutrice jeune et assez «faible»:

Ouais, c'est où qu'il y a eu le tsunami c'est ça? (Azzeddine, 2010, p. 193)

Che, è dove c'è stato lo tsunami? (Azzeddine, 2017, p. 116).

Ce genre de constructions fautives fait d'ailleurs l'objet d'une réflexion métalinguistique de la part de Fairouz, qui se plaint des tournures populaires, argotiques ou triviales employées autour d'elle, en particulier par les membres de sa famille (Azzeddine, 2010, p. 192, p. 201 et encore p. 124-126 et p. 172). La narration d'Azzeddine est émaillée systématiquement de ce genre de réflexions métalangagières, sur lesquelles je reviendrai plus loin. J'ai ensuite mis en place un système de fautes morphosyntaxiques typiques des locuteurs faibles, par exemple, l'usage de «gli» au lieu de «a loro» :

16. Il s'agit de l'édition augmentée et mise à jour du célèbre essai publié en 1987; Sabatini (1985) parle pour sa part de «italiano dell'uso medio » (italien de l'usage moyen). 
Les Noires, elles étaient plus fortes que moi et elles aimaient les Arabes. (Azzeddine, 2009, p. 49)

Le nere erano più forti di me e gli piacevano gli arabi. (Azzeddine, 2011, p. 32).

J'ai par ailleurs eu recours à des tournures largement orales, par exemple, l'usage emphatique du pronom déictique «questo" (celuici) dans la forme "questo qui», ainsi qu'à la forme tronquée de l'adjectif démonstratif «'sto» (questo):

Et même si celui-ci est un peu plus cher (Azzeddine, 2010, p. 14)

$\mathrm{E}$ anche se questo qui è un po' più caro (Azzeddine, 2017, p. 8)

ce Jason (Azzeddine, 2009, p. 27)

'sto Jason (Azzeddine, 2011, 18)

les gourmandes (Azzeddine, 2009, p. 49)

'ste ingorde (Azzeddine, 2011, p. 32)

Dans le souci d'adhérer à la langue des jeunes parlée par les narrateurs et par leur groupe de pairs, j'ai eu recours à l'intensification de préfixes superlatifs «super» et "iper», déjà très présents dans les textes originaux, et dont j'ai parfois souligné la charge juvénile en traduisant par le préfixe italien «stra», diastratiquement marqué :

la littérature anglaise hyper-ancienne (Azzeddine, 2010, p. 188)

la letteratura inglese straantica (Azzeddine, 2017, p. 114)

super beau (Azzeddine, 2009, p. 82)

strabello (Azzeddine, 2011, p. 54)

Au niveau lexical, l'emploi de mots que l'on peut associer au français des cités (Goudaillier, 1997) marque souvent les jeunes locuteurs et contribue à les situer dans un contexte de marginalisation qui se traduit aussi par l'insubordination langagière. Dans ce cas, la difficulté principale pour le traducteur réside dans le fait que la langue italienne ne peut pas compter sur un argot commun ni sur un argot des cités, en préférant jouer sur la variation diatopique. Le dictionnaire historique des langages des jeunes de Renzo Ambrogio et Giovanni Casalegno Scrostati gaggio! (2004) peut aider le traducteur, qui sera en tout cas obligé de tenir compte de l'incidence différente de la variation diastratique en italien. La confrontation 
avec le travail d'autres traducteurs s'avère, à mon avis, vitale ${ }^{17}$. Voici quelques exemples de mes choix traductifs pour rendre des mots argotiques ou verlanisés ${ }^{18}$ :

- «afferrare» pour «piger» tu piges? (Azzeddine, 2009, p. 21)

Hai afferrato il concetto? (Azzeddine, 2011, p. 13)

- «limonare» pour «se rouler des pelles» on se roulait des pelles (Azzeddine, 2009, p. 84) si limonava (Azzeddine, 2011, p. 56)

- «strippare» pour «flipper» J'ai flippé deux fois plus. (Azzeddine, 2009, p. 147) Ho strippato il doppio. (Azzeddine, 2011, p. 99)

- «bonazza» pour «bonne meuf» je me suis dit qu'il n'y avait rien de mieux sur terre que d'être une bonne meuf (Azzeddine, 2009, p. 93)

mi sono detto che sulla terra non c'era niente di meglio che essere una bonazza (Azzeddine, 2011, p. 62)

Si les marques «arg.» et «pop.» donnent les jetons aux traducteurs $^{19}$, que dire de ce langage à clé qu'est le verlan, procédé d'encodage largement employé dans les œuvres littéraires liées au contexte des banlieues, où il se pose comme une sorte de manifeste dans lequel est sédimenté un réseau sémantique et symbolique? La quasi-totalité des langues occidentales ne possèdent pas de langage à métathèse comparable, ce qui rend la traduction particulièrement difficile (Vitali, 2011). À la différence d'autres traducteurs, je n'ai pas

17. Je pense, entre autres, au travail traductionnel et traductologique de Luciana Cisbani (2014). Heureusement, on donne de plus en plus de place à la voix des traducteurs littéraires professionnels, ce qui facilite la mise en réseau d'expériences qui, auparavant, demeuraient souvent privées.

18. Ces exemples, présentés hors contexte, sont à prendre avec précaution : le lecteur comprendra qu'ils ne sont pas à considérer comme des équivalents absolus. Rappelons qu'il n'est d'ailleurs pas possible d'analyser en profondeur une traduction en se limitant à comparer des paires de mots dans la langue source et dans la langue cible, la traduction devant être observée dans son ensemble, car des stratégies de compensation, dont on ne peut évidemment rendre compte ici dans le détail, sont souvent mises en œuvre non seulement au niveau phrastique, mais plus en général au niveau textuel.

19. Je fais ici un clin d'œil au titre de l'article de Chiara Elefante, «Arg. et pop., ces abréviations qui donnent les jetons aux traducteurs-dialoguistes» (2004). 
souhaité créer un verlan artificiel ${ }^{20}$, estimant que cela sonnerait faux à l'oreille d'un locuteur italien, tel un jeu d'enfants plutôt bizarre et complètement détaché de la pratique linguistique réelle. Le résultat d'une telle reproduction, bien que semblable dans sa forme, trahirait l'original bien plus que d'autres solutions. Je connais l'existence, en Italie, de phénomènes langagiers tels que le trancorio, langage à clé basé sur la métathèse syllabique, originaire des provinces de Milan et Brescia ${ }^{21}$. Toutefois, la marginalité de ce langage et l'absence d'un nombre significatif de locuteurs font en sorte qu'il n'a aucun impact sur l'italien dit standard, alors que de nombreux termes du verlan ont été lexicalisés au fil des années, et cela depuis longtemps (meuf > femme en 1981, keuf > flic en 1978, pour ne citer que les exemples les plus connus). En considérant l'ensemble de ces facteurs, l'emploi du trancorio ou de langages fictifs imitant le verlan nétait pas praticable à mon avis $^{22}$. J'ai donc préféré chercher des équivalents fonctionnels dans les couches de la langue parlée, que j'ai émaillée de nombreuses expressions reprises de l'argot des jeunes et du monde du rap, qui vient se métisser de plus en plus ${ }^{23}$. Je pense, par exemple, au mot "tipa», employé par les rappeurs dans le sens de «jeune femme», voire «fiancée», que j'ai utilisé pour traduire «meuf» dans de nombreux cas.

Une autre caractéristique dominante de la langue des cités exploitée dans les romans d'Azzeddine et, plus en général, dans les textes littéraires de notre domaine, est représentée par la forte

20. C'est ce que font notamment Gilda Lombardi et Riccardo Fedriga, traducteurs italiens du premier volet des Chroniques de l'asphalte de Samuel Benchetrit (2005), publié en Italie par Neri Pozza (2007). On pourra consulter, entre autres, le chapitre «Devant la tour»/ «Davanti alla torre».

21. Au sujet de ce langage qui, à ma connaissance, n'a pas encore fait l'objet d'études linguistiques, on peut se référer aux sites Trancorio's blog (s. d.) et Odiopiccolo (2016), qui fournissent des exemples (lobbe>bello (beau), stoqque>questo (ceci), etc.).

22. Du moins, il ne l'est pas aujourd'hui. Il faut également tenir compte de l'évolution du sub-standard et des argots des jeunes. Dans le contexte italien, on consultera à ce sujet le dictionnaire historique de la langue des jeunes déjà cité (Ambrogio et Casalegno, 2004).

23. J'ai donné plusieurs exemples dans un autre article (Vitali,2011).Aujourd'hui, je constate, dans le contexte italien, une mixité culturelle majeure qui se répercute au niveau langagier. Dans le milieu du rap, ressource importante pour les traducteurs qui tentent de reproduire un certain imaginaire linguistique et culturel, je pense notamment à des chanteurs tels que Ghali (d'origine tunisienne), dont les tics de langage dépassent largement la communauté d'origine. 
présence d'écarts diatopiques, notamment des emprunts au darija, basilecte de l'aire maghrébine, ou bien des xénismes, à savoir des mots qui nont pas encore été lexicalisés (Dubois, 1994). Dans le cas des romans d'Azzeddine, on peut classer dans la catégorie des emprunts des termes comme «halal» (Azzeddine, 2010, p. 86), alors que des mots comme "hchouma» (ibid., p. 39), "la honte» en arabe maghrébin, appartiennent à la catégorie des xénismes. En Italie, la communauté maghrébine, moins nombreuse qu'en France, n'a pas eu un impact si important sur la culture nationale, ni d'un point de vue historique ni d'un point de vue social, ni non plus, comme on peut s'en douter, d'un point de vue linguistique ${ }^{24}$. Pour cette raison, les traducteurs qui décident de garder intact le palimpseste linguistique doivent parfois désopacifier certains termes pour le lecteur, par le biais de gloses explicatives ou d'un apparat paratextuel. Pour ma part, j’ai décidé de préserver la polyphonie linguistique, très présente dans La Mecque-Phuket, les personnages appartenant à la communauté maghrébine de France. Dans ce cas, j'ai pu compter sur l'introduction d'un glossaire en fin de texte, une pratique courante chez l'éditeur Il Sirente, spécialisé dans les traductions de textes appartenant à la culture et à la littérature arabe. Des mots comme «hajj» ou «Bismillah» ont pu trouver leur place dans cette annexe. En accord avec l'éditeur, j'ai également respecté la présence dans le texte original de quelques notes en bas de page qui traduisaient des phrases entièrement en arabe, translittérées en alphabet latin. Par exemple :

Ya benti, agi, agi, vissah... ${ }^{1}$

${ }^{1} \mathrm{Ma}$ fille, viens, viens vite... (Azzeddine, 2010, p. 62)

Ya benti, agi, agi, vissah... ${ }^{2}$

${ }^{2}$ Figlia mia, vieni, vieni, presto (Azzeddine, 2017, p. 38) ${ }^{25}$

Bien évidemment, par rapport à la traduction des emprunts et des xénismes, voire de véritables phénomènes de code switching, d'autres solutions peuvent être envisagées, par exemple, l'emprunt

24. On rappelle d'ailleurs que d'après le rapport établi par Bernard Cerquiglini (1999) l'arabe maghrébin fait partie des «langues de France», comme, entre autres, le basque et l'alsacien, le yiddish et le romani. Au sujet du statut de l'arabe maghrébin en France, on pourra également se référer à Barontini (2007).

25. Dans ce passage, le mot «benti», ma fille, employé de manière systématique par la mère de l'héroïne, paraît en italique, ce qui signale que le terme figure dans le glossaire en annexe au roman, selon une pratique établie chez Il Sirente. 
de traits lexicaux, syntaxiques ou phonétiques caractéristiques des langues d'autres communautés immigrées mieux représentées dans la culture cible, dans un souci d'adaptation selon la stratégie du «dialect-for-dialect» exploitée en traduction audiovisuelle. Quoique cette stratégie se prête à maintes critiques (cf. Mével, 2007), elle n'en demeure pas moins possible: pour ne citer qu'un exemple, dans sa traduction allemande des romans d'Azzeddine, Birgit Leib a choisi de puiser avec modération dans la «Kanak Sprak»- un langage revendiqué par les écrivains d'origine turque immigrés en Allemagne et par leurs descendants - pour reproduire le sociolecte parlé dans le milieu des immigrés maghrébins en France (Leib, 2018) $)^{26}$.

Toujours au niveau de la variation diatopique, une autre catégorie, celle du xénolecte (Keil-Sagawe, 2017), un «français cassé» parlé notamment par les parents de l'hérö̈ne du roman La Mecque-Phuket, a constitué un problème traductologique - et idéologique - assez important. J'ai veillé à reproduire ce langage typique des «locuteurs faibles» dans la langue-cible, en ayant recours à l'interlangue des arabophones telle quelle est décrite par Gaetano Berruto (2012), notamment par l'élimination de l'article déterminatif, ou par l'emploi de formes verbales à l'infinitif ou à la troisième personne du présent, comme dans l'exemple qui suit :

Tu croire qu'elle sait? (Azzeddine, 2010, p. 90)

Tu pensa che lei sa? (Azzeddine, 2017, p. 55)

Malgré les résistances de l'éditeur, qui n'aimait pas la reproduction de ce langage et aurait préféré le standardiser, j’ai réussi à le garder, préservant ainsi un trait qui, à mon avis, est l'une des caractéristiques principales de ce roman dont la dynamique interne se déploie autour du conflit générationnel entre les immigrés et leurs descendants nés en France, conflit qui passe par le langage et les usages qu'en font les personnages : alors que les enfants de l'immigration maîtrisent parfaitement le français en tant que locuteurs natifs et connaissent mal l'arabe maghrébin, leurs parents s'expriment dans une interlangue et, très souvent, en darija.

26. Ce procédé est également utilisé dans la traduction suédoise de Faïza Guène. Pour reproduire l'accent et le lexique «banlieusard», la traductrice, Lotta Riad, a recours à l'espagnol sud-américain, la communauté latino-américaine étant bien plus présente en Suède par rapport à la communauté maghrébine (Cf. Aronsson, 2015). 
Pour terminer ce tour d'horizon, il faut mentionner un autre aspect primordial chez Saphia Azzeddine, qui concerne les réflexions métalinguistiques qui constituent l'ossature des deux romans que j'ai traduits. Dans Mon père est femme de ménage, en aidant son père à faire le ménage à la bibliothèque municipale de Saint-Thiers-lèsOsméoles, Polo en profite pour se plonger dans la lecture de chefsd'œuvre de la littérature mondiale. Les romans sont, pour reprendre ses mots, «[s] on salut, [s] a terre promise» (Azzeddine, 2011, p. 103). Chaque semaine, Polo s'efforce d'apprendre un mot nouveau, des mots qu'il emploie pour contraster la misère ambiante qui caractérise son milieu. Fort de ses nouvelles connaissances, le héros corrige systématiquement les membres de sa famille lorsqu'il font de fautes de prononciation, de syntaxe ou de lexique. Une attention semblable au langage se trouve dans La Mecque-Phuket, où Fairouz, comme on l'a vu, prête une attention particulière aux mots et en arrive même à adopter une attitude militante, en invitant chaque jour ses frères et sœurs à enrichir leur maigre vocabulaire. Comme elle l'affirme, elle est «très à cheval sur les mots» (Azzeddine, 2010, p. 16). Toutes ces remarques, dans leur ensemble, guident donc le traducteur et l'invitent à porter une attention exceptionnelle au langage des personnages et à son évolution au fil des pages, car cette évolution témoigne souvent de leur transformation.

Si l'on prend en compte ces aspects dans leur ensemble, il est facile de constater que les difficultés de traduction prennent une valeur exponentielle, car ce que le traducteur doit reproduire n'est pas un sociolecte, mais un idiolecte littéraire créé à partir d'un sociolecte, souvent émaillé de réflexions métacritiques. Comme je l'anticipais, la difficulté principale pour le traducteur réside donc dans le fait que les traits de la «langue des cités» sont un ressort créatif pour Azzeddine - ainsi que pour de nombreux autres écrivains ${ }^{27}$.

\section{Circulation et réception des ouvres}

Les textes, c'est connu, «circulent sans leur contexte» (Bourdieu, 2002, p. 4), ce qui peut engendrer des changements de perspective, voire des méprises et des malentendus. Une fois insérées dans un nouveau contexte, les traductions de romans traitant des banlieues peuvent se heurter à l'incompréhension ou à la mécompréhension,

27. Citons à titre d'exemple Faïza Guène, Habiba Mahany, Mabrouck Rachedi et Rachid Djaïdani. 
ce qui produit un échec ou des difficultés dans la circulation aussi bien des textes que des idées qu'ils véhiculent. D'où la volonté de certains éditeurs de guider au possible la lecture. Cela se reflète, par exemple, dans le choix éditorial de l'un des éléments paratextuels les plus révélateurs : le titre.

Un rapide tour d'horizon dans notre domaine d'étude permet de voir que les traductions comportent souvent des soustitres qui fonctionnent telles des gloses explicatives, en évoquant clairement le contexte des banlieues. Par exemple, Viscéral de Rachid Djaïdani (2007) a été publié en italien avec le titre Viscerale. Un grido dalle banlieue [Viscéral. Un cri des banlieues] (2009). Le petit Malik (Rachedi, 2008) est devenu Malik. Cronache della banlieue [Malik. Chroniques de la banlieue] (Rachedi, 2012), le titre italien oblitérant la référence littéraire, très française et très peu banlieusarde, au Petit Nicolas de Goscinny, au profit d'un renvoi de nature plus sociologique à la marginalité. Le titre du recueil de nouvelles Chroniques d'une société annoncée du collectif Qui fait la France? (2007) a lui aussi été accompagné d'un sous-titre : Cronache di una società annunciata. Racconti dalle banlieue [Chroniques d'une société annoncée. Récits des banlieues] (2009). Lors de la relocalisation dans un nouvel espace culturel, les œuvres, surtout quand il s'agit de premiers romans ou d'auteurs traduits pour la première fois, doivent être contextualisées, et les éditeurs pensent, de toute évidence, qu'un sous-titre explicatif pourra mieux guider le lecteur dans son choix. Cette pratique n'est pas une prérogative de l'espace éditorial italien : on l'observe aussi en Espagne et en Allemagne, notamment, ainsi que dans des pays extra-européens, dont le Japon, où Le petit Malik, pour reprendre l'un des exemples mentionnés ci-dessus, a été traduit par Kôgai shônen Mariku [Malik, garçon de banlieue] ${ }^{28}$ (Rachedi, 2012). En ce qui concerne plus précisément notre étude de cas, les deux éditeurs italiens sont allés à contrecourant : Saphia Azzeddine a été «épargnée» par cette stratégie de positionnement éditorial; aucun sous-titre n'accompagne les titres de ses romans, dont la traduction est, elle-même, littérale.

Un autre ordre d'obstacles concerne la circulation des œuvres. Les données statistiques ne laissent pas de doutes :

28. Pour une réflexion sur les traductions japonaises concernant des textes qui abordent le sujet des banlieues, je renvoie à l'article de Satoshi Udo dans cette livraison de TTR. 
[1]a durée de vie d'un livre en librairie, pour ceux qui arrivent jusqu'aux points de vente, s'est [...] raccourcie en raison de la surproduction et des contraintes imposées par les revendeurs. Dans les chaînes, elle est limitée à trois semaines. (Sapiro, 2012, p. 34)

De plus, le rapport annuel de l'Association Italienne des Éditeurs (Levi, 2018) met en lumière un paradoxe puissant : d'une part, le nombre d'éditeurs augmente; d'autre part, le nombre de lecteurs diminue. L'Italie enregistre en effet un pourcentage de lecteurs très bas comparativement à d'autres pays : en 2016, la moyenne se situait autour de 40,5\% de lecteurs, alors que plusieurs autres pays dépassaient largement $60 \%$. Les statistiques de l'Association Italienne des Éditeurs donnent notamment 62,2\% pour l'Espagne, $68,7 \%$ pour l'Allemagne, $73 \%$ pour les États-Unis, $83 \%$ pour le Canada, $84 \%$ pour la France et $90 \%$ pour la Norvège (ibid.).

Ces données peu encourageantes soulèvent d'autres questions, le problème très concret de la circulation s'imbriquant avec celui de la réception. Comment obtenir un minimum de visibilité dans un marché qui surproduit et où les lecteurs se font de plus en plus rares? L'exemple de Saphia Azzeddine permet d'illustrer la situation. En Italie, on l'a vu, l'œuvre de cette auteure a été publiée dans ma traduction par deux éditeurs : Giulio Perrone a édité le deuxième roman d'Azzeddine, Mon père est femme de ménage (Mio padre fa la donna delle pulizie, 2011), et Il Sirente a publié six ans plus tard son troisième roman, La Mecque-Phuket (La Mecca-Phuket, 2017). Malgré les similitudes entre ces deux romans parus en France chez le même éditeur, les éditeurs italiens ont adopté des stratégies radicalement différentes d'inscription de l'auteure et de son œuvre dans le champ éditorial.

Dans l'esprit de sa collection Hinc, Giulio Perrone a misé sur l'image de la jeune écrivaine et sur son style "poétique et amusant» ("poetico e divertente", comme on peut le lire sur la quatrième de couverture). En suivant (de très loin) la presse française, Perrone a joué également la carte du glamour. Dans une entrevue que j’ai accordée à la revue Vanity Fair (Italie) au sujet de mon travail de traductrice, la journaliste, citant elle-même d'autres sources journalistiques, décrit Azzeddine comme une «écrivaine dans un corps de mannequin» [«una scrittrice in un corpo da modella»] (Pezzino, 2013, n.p.), ce qui est perçu comme une plus-value par rapport au public de la revue (et aurait sans doute été mal perçu 
dans d'autres contextes) ${ }^{29}$. Le second éditeur, Il Sirente, a adopté une stratégie totalement différente, insistant sur les contenus sémantiques et les retombées sociologiques du roman, dont la publication entrait dans le cadre du lancement de la nouvelle collection Altriarabi migrante.

Comme on l'a vu, en tant que traductrice, j'ai traité de la même manière le «corps» de ces deux romans truffés d'argotismes, de xénismes et de mots verlanisés, ajoutant toutefois un glossaire en fin d'ouvrage dans le cas de l'éditeur Il Sirente, selon une pratique établie dans cette maison d'édition. Une fois la traduction terminée, la directrice éditoriale m'a demandé de rédiger une préface, en fonction de mon statut d'universitaire. Il Sirente a ainsi essayé de créer un produit de niche, bien que visant un public non spécialisé. Bref, les deux romans de Saphia Azzeddine sont arrivés entre les mains du public dans des «habits» assez différents, quoique l'objet livre se présentait dans une veste graphique relativement semblable et également soignée (couverture à rabat en quadrichromie, photo d'artiste pour Perrone, illustration conçue ad hoc pour Il Sirente).

Quels ont été les résultats de ces choix éditoriaux si différents pour les ouvrages d'une auteure dont la production est pourtant très homogène et dont les deux romans en question ont été publiés en France par le même éditeur? Comme on peut s'en douter, le choix du positionnement de ces deux traductions dans le champ éditorial italien a entraîné des résultats divergents en termes de reconnaissance de l'auteure et de vente des livres. Dans le premier cas, l'écrivaine a obtenu de la visibilité et un petit succès médiatique; des comptes rendus et des entrevues dans des magazines (surtout féminins) et des quotidiens à grand tirage (par exemple $\mathrm{Il}$ Corriere della sera et La Repubblica) qui portaient surtout sur la voix drôle, originale et fraîche de l'auteure, lui ont été consacrés. Dans le second cas, la traduction est passée presque inaperçue, même si l'écrivaine pouvait déjà compter sur un petit capital, pour ainsi dire, auprès du public italien. La stratégie adoptée par Il Sirente nétait pas gagnante pour favoriser la circulation du roman : au moment de sa parution,

29. Je tiens à signaler qu'en Italie l'auteure ne jouit aucunement du succès médiatique qui la caractérise en France, où elle a été un sujet de la "presse people», notamment pour son ancienne relation avec l'acteur Jamel Debbouze. Le public italien n'a aucune connaissance de ces aspects. De plus, le film que l'écrivaine a tiré de son roman Mon père est femme de ménage, avec François Cluzet à l'affiche, n'est jamais sorti dans les salles italiennes. 
en mars 2017, la question des banlieues ne faisait pas la une, et bien que la situation de l'immigration et surtout des «deuxièmes générations» soit désormais devenue urgente en Italie, le lien avec ce qui se passe (ou ce qui s'est passé) dans d'autres pays européens ne semble pas si évident. De plus, la publication un mois plus tard par le même éditeur de la traduction italienne d'Un homme, ça ne pleure pas (Un uomo non piange mai) de Faïza Guène (2017), qui jouit d'un capital symbolique bien supérieur à celui d'Azzeddine et qui pouvait remplir la même fonction dans un débat d'idées au sujet des banlieues et des deuxièmes générations d'origine maghrébine, a vite éclipsé La Mecca-Phuket.

Une recherche dans le catalogue central du réseau des bibliothèques italiennes (Sistema Bibliotecario Nazionale) montre que le roman d'Azzeddine paru chez Giulio Perrone est présent dans 43 bibliothèques alors que celui publié par Il Sirente n'est présent que dans 2 bibliothèques ${ }^{30}$. Cela conforte l'idée que l'impact d'une œuvre - dans notre cas d'une traduction - est souvent dicté par les politiques éditoriales ainsi que par la résonnance médiatique que peuvent engendrer la figure d'un auteur, une question d'actualité ou une situation d'urgence comme la crise des banlieues. La flambée des traductions d'ouvrages concernant les cités françaises après les émeutes de l'automne 2005 en témoigne, et cela, non seulement en Italie mais dans de nombreux pays. La stratégie d'Il Sirente visant la création d'une collection marquée par un engagement social ainsi qu'un investissement sur des titres de backlist pourrait cependant avoir besoin de plus de temps pour s'épanouir pleinement et s'avérer gagnante pour la reconnaissance des auteurs, au-delà des retombées marketing, sur la moyenne et longue durée.

Par ailleurs, alors que certains comptes rendus des romans d'Azzeddine parus en Italie abordent la question de la jeunesse défavorisée, pratiquement aucun ne se penche sur la question religieuse $^{31}$, même si celle-ci fait partie des thèmes traités dans $L a$

30. Il faudrait sans doute considérer le facteur temps et réexaminer ces aspects sur la moyenne et longue durée. Cependant, si l'on tient compte de la quantité exceptionnelle de livres publiés chaque mois, il est peu probable qu'une bibliothèque achète un roman contemporain longtemps après sa période de publication (et donc sa période de visibilité maximale), sauf si un nouveau roman d'un auteur est particulièrement apprécié et vient mettre en lumière ses romans antérieurs.

31. À l'exception de L'Avvenire, quotidien d'inspiration catholique qui a publié un article-entretien portant en partie sur cette question (Michelucci, 2017). 
Mecque-Phuket. Sur ce plan, si l'on compare l'Italie à l'Allemagne et l'Espagne, les seuls autres pays où Azzeddine est publiée à ce jour, on remarque que les journalistes dans ces deux pays semblent soulever plus de questions en ce sens ${ }^{32}$. Ce n'est qu'un exemple de la manière dont les textes d'un même auteur peuvent être perçus selon les contextes et le positionnement de l'éditeur.

\section{En guise de conclusion}

Comme on a pu l'observer grâce à ces quelques réflexions, la nature des traductions est déterminée par une série de facteurs et de conditions dont les traductologues doivent nécessairement tenir compte, sans se limiter à l'analyse contrastive pour cautionner l'ancien adage "traduttore, traditore». De fait, toute traduction publiée s'inscrit dans une démarche éditoriale précise dont elle est le produit et qui est régie par les lois du marché de l'édition. Ces lois sont parfois dures; elles témoignent notamment d'un rapport de force entre langues et cultures tenues comme "majeures» et comme "mineures", rapport qui détermine la circulation internationale des œuvres (Sapiro, 2008 et 2012) ainsi que le choix d'un positionnement précis dans le champ éditorial. Cela est d'autant plus vrai pour les œuvres d'écrivains dits «de banlieue», qui se positionnent normalement aux marges de l'échiquier éditorial et qui, généralement, n’en gagnent le centre que lorsqu'un évènement social attire l'attention des médias.

Les solutions traductives que j'ai proposées dans cette étude de cas sont à considérer dans leur contexte, en prenant aussi en compte que toute analyse traductologique reflète un état de la recherche et doit nécessairement être considérée comme provisoire. Ce n'est pas un hasard si le traducteur italien Daniele Petruccioli compare la traduction à l'exécution musicale, en reprenant la métaphore texte/ partition chère à Paul Ricœur. Pour Petruccioli, le traducteur est un

32. En Allemagne, Azzeddine est publiée par Klaus Wagenbach, l'un des éditeurs les plus influents du point de vue culturel. Fondée à Berlin Ouest en 1965, cette maison d'édition a dès le départ manifesté son intérêt politique par la publication d'écrivains et intellectuels socialistes. Son catalogue, très cohérent, reflète son ancrage idéologique. Cette maison se distingue par sa démarche engagée, voire militante, qui lui donne un capital symbolique particulier à l'intérieur de l'espace éditorial. En Espagne, Azzeddine est publiée par deux éditeurs : Demipage et Grijalbo, éditeur mexicain ayant une filiale en Espagne. Une analyse rapide de la réception espagnole d'Azzeddine fait émerger, dans son ensemble, une attention à la condition féminine associée en particulier à la religion islamique. 
interprète du texte source (la partition), et cela devrait être clair pour le lecteur averti tout comme ce l'est pour le mélomane : une sonate de Beethoven sera différente selon le musicien qui la joue, et celui qui l'écoute sera bien conscient que ce n'est pas Beethoven qui est en train de la jouer (2012, p. 71). De la même manière, des traducteurs différents traduiront le même texte de manière différente, selon leur sensibilité ainsi que selon le contexte. Petruccioli va jusqu'à affirmer qu'un même traducteur aborderait de manière différente un même texte dans le temps, parce que les conditions auront changé, que la langue aura évolué ou, tout simplement, parce que diverses options existent.

À cela, il faut nécessairement ajouter que les romans des auteurs traitant de la banlieue, et notamment ceux de Saphia Azzeddine, invitent à sortir de ce manichéisme qui oppose traducteurs sourciers et ciblistes, car, comme de nombreux spécialistes l'ont observé au fil des dernières décennies (voir par exemple Bandia, 2003), les oppositions rigoureusement binaires typiques d'un certain discours traductologique s'appliquent mal à des auteurs dont l'œuvre est caractérisée par l'hybridité, le multiculturalisme et l'hétéroglossie.

Petruccioli souhaite à juste titre un éveil de la conscience critique des éditeurs et des lecteurs. Il faut reconnaître que le travail de nombreuses maisons d'édition indépendantes italiennes est appréciable en ce sens, ce qui se reflète entre autres dans la place accordée au traducteur, de la proposition des textes jusqu'à la publication de l'ouvrage, parfois accompagné d'un apparat paratextuel. Celui qui lit peut bien évidemment ignorer cette voix placée en marge du texte, mais sa présence rassure a contrario les lecteurs les plus exigeants, qui peuvent comprendre par qui et de quelle manière «la sonate» est interprétée. C'est ce genre de prise de conscience que Petruccioli souhaite au lecteur des textes traduits. Nous ne pouvons que partager ce désir.

\section{Références}

Aronsson, Mattias (2015). «Faïza Guène chez les Vikings: quelques réflexions à propos de la traduction suédoise d'un discours argotique et "beur"». Moderna språk, 109, 1, p. 29-49.

Azzeddine, Saphia (2009). Mon père est femme de ménage. Paris, Léo Scheer. Azzeddine, Saphia (2010). La Mecque-Phuket. Paris, Léo Scheer.

Azzeddine, Saphia (2011). Mio padre fa la donna delle pulizie. Trad. Ilaria Vitali. Rome, Giulio Perrone. 
Azzeddine, Saphia (2017). La Mecca-Phuket. Trad. Ilaria Vitali. Rome, Il Sirente.

Berman, Antoine (1985). «La traduction et la lettre, ou l'auberge du lointain». In Les tours de Babel. Mauvezin, Trans-Europ-Repress, p. 35-150.

Bandia, Paul F. (2003). «Postcolonialism and Translation: The Dialectic between Theory and Practice». Linguistica Antverpiensia, New Series, 2, p. 129-141.

Barontini, Alexandrine (2007). "Valorisation des langues vivantes en France : le cas de l'arabe maghrébin». Le Français aujourd'hui, 158, 3, p. 20-27.

Berruto, Gaetano (2012). Sociolinguistica dell'italiano contemporaneo. Roma, Carocci.

Bertucci, Marie-Madeleine et Daniel Delas, dir. (2004). Français des banlieues, français populaire? Amiens, Ancrage.

Bonn, Charles et Coordination internationale des chercheurs sur les littératures du Maghreb (1998). Limag. Littératures du Maghreb (Algérie, Maroc, Tunisie, 1806-2015). Disponible à <http://www.limag.refer. org/> [consulté le 28 juillet 2018].

Bouillot, Françoise (2010). "Theory et bricolage: confessions d'une traductrice». Revue française d'études américaines, 126, p. 82-91.

Bourdieu, Pierre (2002). «Les conditions sociales de la circulation internationale des idées ». Actes de la recherche en sciences sociales, 145, p. 3-8.

Campanelli, Chiarastella (2015). «Presentazione della collana Altriarabi migrante» In J. Ruillier, Se ti chiami Mohamed. Rome, Il Sirente.

Carpenter, Juliet et Christina Horvath (2014). Voices and Images from the Banlieue/Voix et images de la banlieue. Oxford, Banlieue Network, Oxford Brookes University.

Cerquiglini, Bernard (1999). Les Langues de France : rapport au ministre de l'Éducation nationale, de la Recherche et de la Technologie et à la ministre de la Culture et de la Communication. Disponible à <http://www.dglf. culture.gouv.fr/> [consulté le 7 septembre 2018].

Cisbani, Luciana (2014). "Quella mala lingua che traduce l'argot». Argotica, 1, 3, pp. 215-220.

Collier, Anne-Claire (2015). "Traduire les études postcoloniales en France». Convergences francophones, 2, 1, p. 56-71.

Conein, Bernard et Françoise Gadet (2000). « Français populaire? Français des banlieues?» In F. Aitsiselmi, dir. Black, Blanc, Beur Youth: Language and Identity in France. Bradford, University of Bradford Department of Modern Languages.

Djaidani, Rachid (2007). Viscéral. Paris, Seuil.

Djaidani, Rachid (2009).Viscerale. Un grido dalle banlieue. Trad. Ilaria Vitali. Roma, Giulio Perrone.

Dubois, Jean et al. (1994). Dictionnaire de linguistique. Paris, Larousse. 
Elefante, Chiara (2004). «Arg. et pop., ces abréviations qui donnent les jetons aux traducteurs-dialoguistes». Meta, 49, 1, p. 193-207.

Elefante, Chiara (2015). «La littérature urbaine à l'épreuve de la traduction en italien. Une analyse socio-édito-traductologique». Repères-Dorif, 8. Disponible à <http://www.dorif.it/ezine/ezine_articles.php?art_ id=241> [consulté le 20 août 2018].

Goudaillier, Jean-Pierre (1997). Comment tu tchatches! Dictionnaire du français contemporain des cités. Paris, Maisonneuve et Larose.

Guène, Faïza (2014). Un homme, ça ne pleure pas. Paris, Fayard.

Guène, Faïza (2017). Un uomo non piange mai. Trad. Federica Pistono. Roma, Il Sirente.

Hargreaves, Alec G. (2014). «De la littérature «beur» à la littérature de «banlieue»: des écrivains en quête de reconnaissance». Africultures, 97, p. 144-149.

Horvath, Christina (2016). «Écrire la banlieue dans les années 2000-2015 ». In B. Wallon, dir. Banlieues vues d'ailleurs. Les Essentiels d'Hermès. Paris, CNRS Éditions, p. 47-68.

Keil-Sagawe, Regina (2017). «La Khadrature du Cercle : ou comment traduire les auteurs du Maghreb pour le public allemand?». In A. Nouss, C. Pynçonnat et F. Rinner, dir. Littératures migrantes et traduction. Aixen-Provence, Presses universitaires de Provence, p. 187-197.

Lapeyronnie, Didier (2005). «La banlieue comme théâtre colonial, ou la fracture coloniale dans les quartiers». In P. Blanchard, N. Bancel et S. Lemaire, dir. La fracture coloniale. La société française au prisme de l'héritage colonial. Paris, La Découverte, p. 209-218.

Leib, Birgit (2018). «Entretien personnel inédit». Réalisé le 10 mars 2018. Levi, Ricardo Franco (2018). «Il mercato del libro in Italia nel 2017». Associazione Italiana Editori [Association Italienne des Éditeurs]. Disponible à <http://www.aie.it/Portals/_default/Skede/Allegati/ Skeda105-4104-2018.1.25/2017_01_26_Mercato-2017-DA\%20 CARICARE.pdf?IDUNI=tlnnqco5yshhgktw13 txw4b19613> [consulté le 20 août 2018].

Mével, Pierre-Alexis (2007). "The Translation of Identity: Subtitling the Vernacular of the French Cité». MHRA Working Papers in the Humanities, 2, p. 49-56.

Michelucci, Riccardo (2017). «La scrittrice Saphia Azzedine : banlieue, la rabbia non è islam», L'Avvenire, 8 avril. Disponible à <https:// www.avvenire.it/agora/pagine/banlieue-saphia-azzedine> [consulté le 29 juillet 2018].

Odiopiccolo (2016). «Il trancorio, storia di una lingua tutta bresciana». Odiopiccolo, 30 août. Disponible à <http://www.odiopiccolo.com/new_ site/il-trancorio-storia-di-una-lingua-tutta-bresciana/> [consulté le 21 août 2018]. 
Paquot, Thierry, dir. (2008). Banlieue. Une anthologie. Lausanne, Presses polytechniques et universitaires romandes.

Petruccioli, Daniele (2012). Falsi d'autore. Macerata, Quodlibet.

Pezzino, Laura (2013). "Lost in Translation - Percorsi». Vanity Fair, 25 mars. Disponible à <http://bookfool.vanityfair.it/2013/03/25/lostin-translation-percorsi/?refresh_ce=> [consulté le 29 juillet 2018].

Qui fait la France? (2007). Chroniques d'une société annoncée. Paris, Stock.

Qui fait la France? (2009). Cronache di una società annunciata. Racconti dalle banlieue. Trad. Ilaria Vitali. Roma, Stampa Alternativa.

Rachedi, Mabrouck (2008). Le petit Malik. Paris, Lattès.

Rachedi, Mabrouck (2012). Malik. Cronache della banlieue. Trad. Clizia Cevasco. Milano, Leone Editore.

Rachedi, Mabrouck (2012). Kôgai shônen Mariku [Malik, garçon de banlieue]. Trad. Nakajima Saori. Tokyo, Shûeisha.

Reeck, Laura (2011). Writerly Identities in Beur Fiction and Beyond. Lanham, Lexington Books.

Sabatini, Francesco (1985). "L'italiano dell'uso medio: una realtà tra le varietà linguistiche italiane. In G. Holtus et E. Radtke, dir. Gesprochenes Italienisch in Geschichte und Gegenwart. Tübingen, Gunter Narr Verlag, p. 154-184.

Sapiro, Gisèle (2008). Translatio. Le marché de la traduction en France à l'heure de la mondialisation. Paris, CNRS.

Sapiro, Gisèle (2012). «Les obstacles économiques et culturels à la traduction». In G. Sapiro, dir. Traduire la littérature et les sciences humaines. Conditions et obstacles. Paris, La documentation française, p. 25-53.

Syndicat national de l'édition de France, «Les cessions de droit à l'international». Disponible à <https://www.sne.fr/economie/les-cessions-dedroit-a-linternational/> [consulté le 10 septembre 2018].

Trancorio's blog (s.d.). Disponible à <http://trancorio.blogspot.com> [consulté le 21 aout 2018].

Turpin, Béatrice (2012). Discours et sémiotisation de l'espace. La représentation de la banlieue et de sa jeunesse. Paris, L'Harmattan.

Vlahov S. et S. Florin (1986). Neperovodimoe v perevode. Moscou, Vysšaja škola.

Venuti, Lawrence (1998). The Scandals of Translation: Towards an Ethics of Difference. London, New York, Routledge.

Vitali, Ilaria (2011). «Les écrivains beurs comme traducteurs?». In I. Vitali, dir. Intrangers, II, Littérature beur : de l'écriture à la traduction. Louvainla-Neuve, Academia Bruylant; Paris, L'Harmattan.

Vitali, Ilaria (2014). La nebulosa beur. Scrittori di seconda generazione tra spazio francese e letteratura-mondo. Bologna, Odoya/I libri di Emil.

Vitali, Ilaria (2015). «En long, en large et en verlan : l'emploi littéraire du français des cités». In F. Impellizzeri, dir. Les variations linguistiques 
dans la littérature et le cinéma contemporains. Paris, Classiques Garnier, p. 19-33.

Waberi, Abdourahman A. (1998). «Les enfants de la postcolonie. Esquisse d'une nouvelle génération d'écrivains francophones d'afrique noire». Notre Librairie, 135 , p. 8-15.

Wallon, Bernard, dir. (2016). Banlieues vues d'ailleurs. Paris, CNRS Éditions.

Ilaria Vitali

Dipartimento di Studi Umanistici

Università di Macerata

Macerata, Italia

ilaria.vitali@unimc.it 